\title{
Associations of day of week and time of day of ICU admission with hospital mortality in Malawi
}

\author{
Meghan Prin' (1), Ruoyu $\mathbf{J i}^{2}$, Clement Kadyaudzu ${ }^{3}$, Guohua Li ${ }^{4,5}$ \\ and Anthony Charles ${ }^{6}$
}

\begin{abstract}
This prospective cohort study evaluated the associations of day and time of admission to the Intensive Care Unit (ICU) with hospital mortality at a referral hospital in Malawi, a low-income country in sub-Saharan Africa. Patients admitted to the ICU during the day (08:00-16:00) were compared to those admitted at night (16:0I-07:59); patients admitted on weekdays (Monday-Friday) were compared to admissions on weekends/holidays. The primary outcome was hospital mortality. Most patients were admitted during daytime $(56 \%)$ and on weekdays $(72 \%)$. There was no difference in mortality between night and day admissions $(58 \%$ vs. $56 \%, P=0.8828$; hazard ratio $[\mathrm{HR}]=1.09,95 \%$ confidence interval $[\mathrm{Cl}=0.82-\mathrm{I} .44, P=0.56 \mathrm{I})$ or weekend/holiday versus weekday admissions $(56 \%$ vs. $57 \%, P=0.90 \mathrm{II} ; \mathrm{HR}=0.87,95 \%$ $\mathrm{Cl}=0.62-I .2 \mathrm{I}, P=0.4 \mid 33)$. No interaction between time and day was found. These results may be affected by high overall hospital mortality.
\end{abstract}

\section{Keywords}

Outcomes, global health, Intensive Care Unit organisation

\section{Introduction}

Critically ill patients benefit from timely, quality intensive care medicine. Delays or deficiencies in care secondary to organisational factors have been associated with poor patient outcomes. These factors include the availability of dedicated intensive care specialist physicians (as opposed to general physicians), ${ }^{1}$ 'closed' versus 'open' clinical management styles, ${ }^{2,3}$ and nursing staff patterns. ${ }^{4}$ Regardless of the specific operational organisation of an intensive care unit (ICU), some factors such as staffing and workload are expected to vary over the course of each day, week and season.

The timing of admission to the ICU (e.g. daytime versus night-time, weekend versus weekdays) has been evaluated in multiple studies, ${ }^{5-11}$ but not often in a lowincome country or in the region of sub-Saharan Africa. Research that informs these questions has been cited as a path forward in global health critical care medicine. ${ }^{12}$ Organisational factors affecting patient care are particularly important questions to address and optimise in the African region, because availability of ICU beds here is generally only 1 per million population or less ${ }^{13}$ and physician oversight in the ICU (generalist or specialist) is rare. A recent meta-analysis demonstrated that an increased odds ratio for hospital mortality among weekend versus weekday ICU admissions was limited to North American studies, which suggests that this relationship may be modified by geographic setting and healthcare system. ${ }^{14}$ The aim of our study was to evaluate the association of time-of-day and day-of-

\footnotetext{
'Assistant Professor, Department of Anesthesiology, University of Colorado Medical Center, Aurora, CO, USA

${ }^{2}$ Department of Biostatistics, Columbia University Mailman School of Public Health, New York, NY, USA

${ }^{3}$ Clinical Officer, Department of Anesthesiology, Kamuzu Central Hospital, Lilongwe, Malawi

${ }^{4}$ Professor, Department of Anesthesiology, Columbia University College of Physicians and Surgeons, New York, NY, USA

${ }^{5}$ Professor, Department of Epidemiology, Columbia University Mailman School of Public Health, New York, NY, USA

${ }^{6}$ Professor, Department of Surgery, University of North Carolina at Chapel Hill, Chapel Hill, NC, USA
}

\section{Corresponding author:}

Meghan Prin, Department of Anesthesiology, University of Colorado, I240I E. 17th Avenue, 7th Floor, Aurora, CO 80045, USA.

Email: meghan.prin@cuanschutz.edu 
week on hospital mortality among patients admitted to the ICU in a public referral hospital in Malawi.

\section{Methods}

This was a prospective, observational cohort study of patients admitted to the ICU of Kamuzu Central Hospital (KCH) in Lilongwe, Malawi, from September 2016 to July 2018. The timing of the study recruitment was based on funding limitations; no power calculations were undertaken. The study protocol was registered at researchregistry.com under protocol 4330 . It was developed a priori and approved by the National Health Sciences Research Council of Malawi and the Institutional Review Boards of both American universities with which the study was affiliated, and the requirement for written informed consent was waived by all. The results are reported in line with the Strengthening the Reporting of Cohort Studies in Surgery (STROCSS) criteria. ${ }^{15}$

Malawi is a country in southern Africa with a population of 18 million people, a life expectancy of 63.8 years and a Human Development Index rank of 170 out of 187 countries. ${ }^{16}$ It is the fourth poorest country in sub-Saharan Africa. ${ }^{17} \mathrm{KCH}$ is a referral hospital in the central region of Malawi with a catchment area of approximately 5 million. The ICU at $\mathrm{KCH}$ is a five-bed unit which offers a 1:1 nurse-to-patient ratio during the day and a 1:2 or higher nurse-to-patient ratio at night. Resources include continuous non-invasive vital sign monitoring, mechanical ventilation with a titratable fraction of inhaled oxygen, and intravenous medication infusions. Haemodialysis is available within the hospital campus. Clinical care in the ICU is directed by clinical officers in Anaesthesiology; exactly one clinical officer is on duty in the ICU at all times to oversee patient care. Although physicians from other services (e.g. Surgery, Obstetrics) consult on ICU patients, no physicians are on staff in this unit and no staff members have consultant-level expertise in intensive care medicine.

The data were collected prospectively by study staff trained in ICU data abstraction. Variables collected included the day and time of ICU admission, vital signs and laboratory measurements at the time of ICU admission, treatments utilised in ICU (e.g. mechanical ventilation), the date of ICU discharge and the hospital discharge date. Laboratory data collected included only those commonly measured by local standards and available in the hospital laboratory. For this study, this included a complete blood count, electrolytes, point-of-care malaria status and point-of care human immunodeficiency virus (HIV) status.

All adult patients admitted to the study ICU were eligible for inclusion. Exclusion criteria included age $\leq 15$ years, readmission to ICU during the same hospitalisation, and missing primary exposure and/or multiple key data points. We performed analyses with and without patients admitted to ICU for a reported severe head injury, because this group is known to have very high mortality. ${ }^{18}$ The analysis excluding patients with severe head injury is included in the Supplemental Materials.

The cohort was divided into groups based on the time of ICU admission. We split each day based on shift hours at the study hospital (i.e. daytime [DT] hours are a period of more nurses with a 1:1 nurseto-patient ratio, while the night-time [NT] hours generally have less staff). DT was defined as 08:00-16:00 and NT as 16:01-07:59 the next day. The days of the week were then grouped as weekdays (Monday-Friday) or weekends (Saturday, Sunday and public holidays as listed in Appendix A). This yielded the following

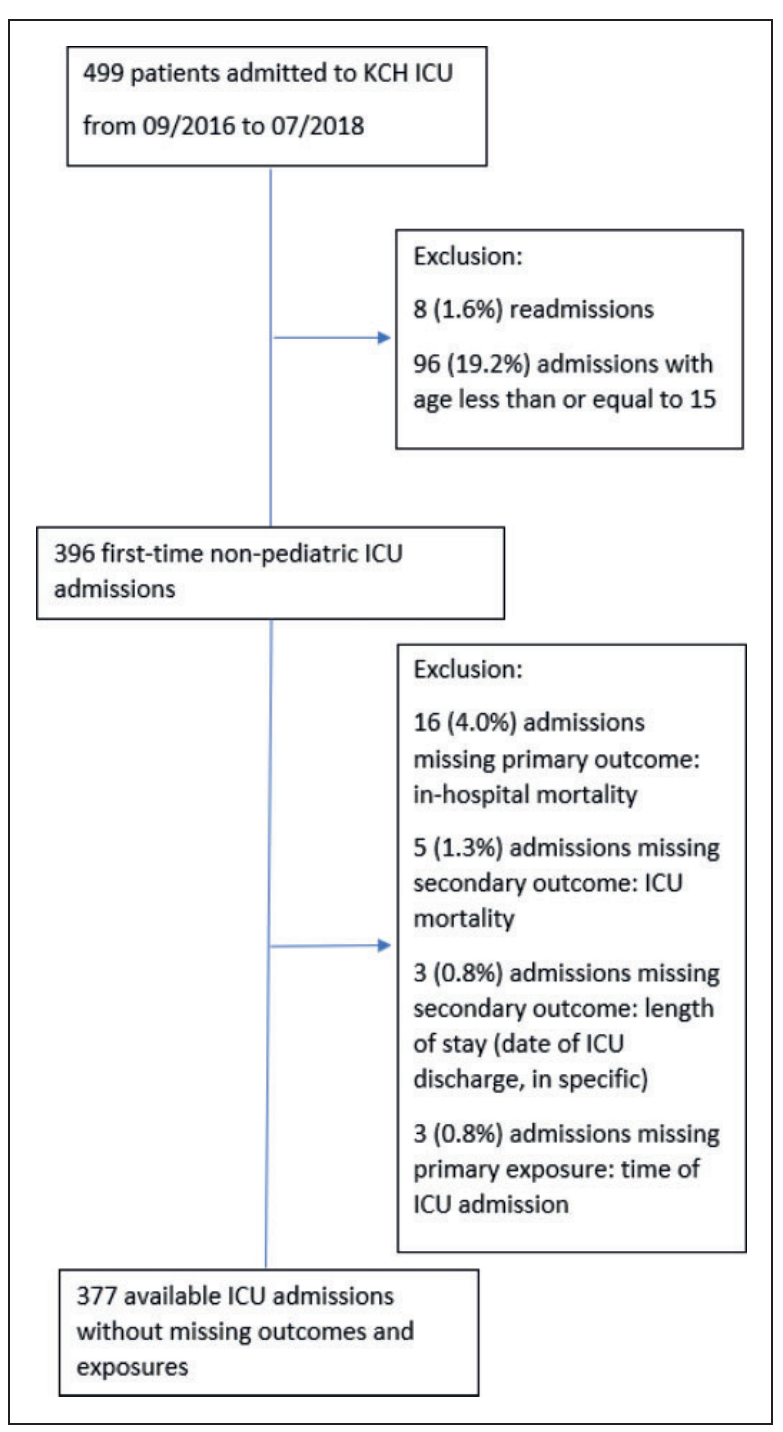

Figure I. Flow diagram for cohort. 
Table I. Clinical characteristics and hospital course for study cohort, stratified by time of day of ICU admission.

\begin{tabular}{|c|c|c|c|c|}
\hline Variable & Total & Daytime & Night-time & $P$ value \\
\hline Total ICU admissions & 377 & $210(55.7)$ & $167(44.3)$ & \\
\hline Age (years) & $3 I(23-4 I)$ & $32(23-42)$ & $30(23-39)$ & $0.3086 *$ \\
\hline Sex & & & & $0.3317^{\dagger}$ \\
\hline Female & $196(52.0)$ & $104(49.5)$ & $92(55.1)$ & \\
\hline Male & $|8|(48.0)$ & $106(50.5)$ & $75(44.9)$ & \\
\hline \multicolumn{5}{|c|}{ Hospital course preceding ICU admission } \\
\hline Time in hospital before ICU (days) & I (0-2) & I $(0-2.75)$ & $I(0-I)$ & $0.02896 *$ \\
\hline Hospital location before ICU admission & & & & $0.1971^{\dagger}$ \\
\hline Emergency room & $71(18.8)$ & $39(18.6)$ & $32(19.2)$ & \\
\hline HDU & $91(24.1)$ & $59(28.1)$ & $32(19.2)$ & \\
\hline Operating room & $180(47.7)$ & 91 (43.3) & $89(53.3)$ & \\
\hline Outside hospital & $12(3.2)$ & $6(2.9)$ & $6(3.6)$ & \\
\hline Ward & $23(6.1)$ & $15(7.1)$ & $8(4.8)$ & \\
\hline Recent surgery in hospital course & $238(63.1)$ & $122(58.1)$ & $116(69.5)$ & $0.03041^{\dagger}$ \\
\hline Cardiac arrest before ICU & $45(11.9)$ & $26(12.4)$ & $19(11.4)$ & $0.8897^{\dagger}$ \\
\hline ICU admitting service & & & & 0.0004517 \\
\hline General surgery & $234(62.1)$ & $130(6 \mid .9)$ & $104(62.3)$ & \\
\hline OBGyn & $98(26.0)$ & $44(21.0)$ & $54(32.3)$ & \\
\hline Medicine & 45 (II.9) & $36(17.1)$ & $9(5.4)$ & \\
\hline \multicolumn{5}{|c|}{ Vital signs and assessments at admission to ICU } \\
\hline Total MEWS & $7(5-8)$ & $6(5-8)$ & $7(5-8)$ & $0.4391 *$ \\
\hline Heart rate (bpm) & $117(98-134)$ & $115(96-132)$ & $119(100.25-136.75)$ & $0.2488^{*}$ \\
\hline Respiratory rate & $19(14-25)$ & $19(14-25)$ & $18.5(14-25)$ & $0.7166^{*}$ \\
\hline$M A P^{\S}(\mathrm{mmHg})$ & $88.77(72.27-104.86)$ & $91.25(74.09-106.01)$ & $86.46(71.03-104.53)$ & $0.2798 *$ \\
\hline Temperature $\left({ }^{\circ} \mathrm{C}\right)$ & $36(34.7-37)$ & $36(35-37)$ & $36(34-37)$ & $0.2772 *$ \\
\hline Pulse oximetry value & $97(93-99)$ & $97(91-99)$ & $98(95-99)$ & $0.0556 \mathrm{I} *$ \\
\hline Altered mental status & $313(83.7)$ & $176(84.2)$ & $137(83.0)$ & $0.8683^{\dagger}$ \\
\hline Infection suspected & $207(54.9)$ & $131(62.4)$ & $76(45.5)$ & $0.00155^{\dagger}$ \\
\hline HIV-positive & $4 \mid(12.9)$ & $24(13.6)$ & $17(12.1)$ & $0.8378^{\dagger}$ \\
\hline Malaria & $33(11.5)$ & $25(15.2)$ & $8(6.5)$ & $0.03643^{\dagger}$ \\
\hline \multicolumn{5}{|l|}{ Clinical course in ICU } \\
\hline Provision of mechanical ventilation & $364(96.6)$ & $203(96.7)$ & I6I (96.4) & $\sim l^{\dagger}$ \\
\hline Length of mechanical ventilation (days) & $2(I-6)$ & $3(I-6)$ & $2(I-5)$ & $0.589 *$ \\
\hline Length of stay in ICU (days) & $3(I-6)$ & $3(I-7)$ & $3(I-5)$ & $0.3588^{*}$ \\
\hline ICU mortality & 191 (50.7) & $104(49.5)$ & $87(52.1)$ & $0.6947^{\dagger}$ \\
\hline Hospital mortality & $214(56.8)$ & $118(56.2)$ & $96(57.5)$ & $0.8828^{\dagger}$ \\
\hline
\end{tabular}

Values are given as $\mathrm{n}(\%)$ or median (IQR).

*Wilcoxon rank-sum test (non-parametric equivalent for two-sample $t$ test) for comparisons of continuous variables.

${ }^{\dagger}$ Chi-squared test for homogeneity/independence of categorical variables: Assumptions: (i) independent groups; (ii) no expected cell counts are 0 and no more than $20 \%$ of cells have an expected count $<5$.

$\S$ MAP is calculated using the following equation: $0.66 \times($ diastolic BP $)+0.33 \times($ systolic BP).

BP, blood pressure; bpm, beats per minute; ICU, Intensive Care Unit; IQR, interquartile range; HDU, high-dependency unit; HIV, human immunodeficiency virus; MAP, mean arterial pressure; MEWS, Modified Early Warning Score; OBGyn, Obstetrics and Gynaecology.

non-exclusive groups for comparison: patients admitted at night; patients admitted during the day; patients admitted on weekdays; and patients admitted on weekends or public holidays. We sought to test two hypotheses: (1) hospital mortality differs between weekday versus weekend/public holiday ICU admissions; and
(2) hospital mortality differs between DT versus NT ICU admissions. Given our anticipated limited sample size, we did not seek to compare patients on a more granular level.

We summarised the clinical traits of the entire cohort and then compared the groups. We performed 
univariate analyses to identify potential confounders of the relationship between timing of admission to the ICU and hospital mortality. Potential confounders were identified using a $P$ value cut-off of 0.2 . Special consideration was given to confounders identified a priori: severity of illness based on the Malawi Intensive Care Mortality Evaluation (MIME) model ${ }^{19}$ (age, sex, admitting service, systolic pressure at ICU admission, altered mental status at ICU admission and fever during the ICU course); the number of hospital days before ICU admission; and cardiopulmonary resuscitation before ICU admission. The primary outcome was hospital mortality.

We conducted survival analyses for the primary outcome following the example of Arulkumaran et al..$^{5}$ : (1) an unadjusted model of weekday versus weekend/ public holiday, which used weekday as the reference; (2) an unadjusted model of time-of-day, which used DT as the reference, as well as an assessment of the interaction between time-of-day and day-of-week (see Supplementary Analyses). The estimated KaplanMeier survival curves were compared using the logrank test, with right-censoring at hospital discharge. We then performed multivariable survival analyses. For the adjusted models, we built Cox proportional hazard regression models. The regression models initially included variables from our univariate analyses: time and day of admission to the ICU; age; sex; admitting service; systolic blood pressure; heart rate; respiratory rate; altered mental status or suspicion of infection at ICU admission; fever during the ICU course; surgery within the index hospitalisation; and the length of hospital stay before admission to the ICU. We used backward selection to develop more parsimonious models based on these results. Finally, we plotted the adjusted Kaplan-Meier survival curves for hospital mortality. For every patient in the dataset, a prediction for hospital survival was made. Subpopulations of interest were balanced with respect to covariates to keep distributions similar in the whole dataset. The balancing was performed in the following way: (1) for each subpopulation, a logistic regression model was created to model the odds of being in the subpopulation given the other covariates; (2) reverse probabilities of belonging to a specific subpopulation were used as weights in the Cox model; (3) the Cox model was refitted with weights; and (4) expected survival curves were calculated for each subpopulation based on a refitted weighted model. The predictions were averaged for subpopulations after rebalancing.

Continuous data are presented as medians with interquartile ranges (IQR), categorical data as number and proportion of the total. Model output is described with hazard ratios (HR) and 95\% confidence intervals (CI). Statistical significance was set at 0.05 . Statistical analyses were conducted using $\mathrm{R}$ version 3.5.1.

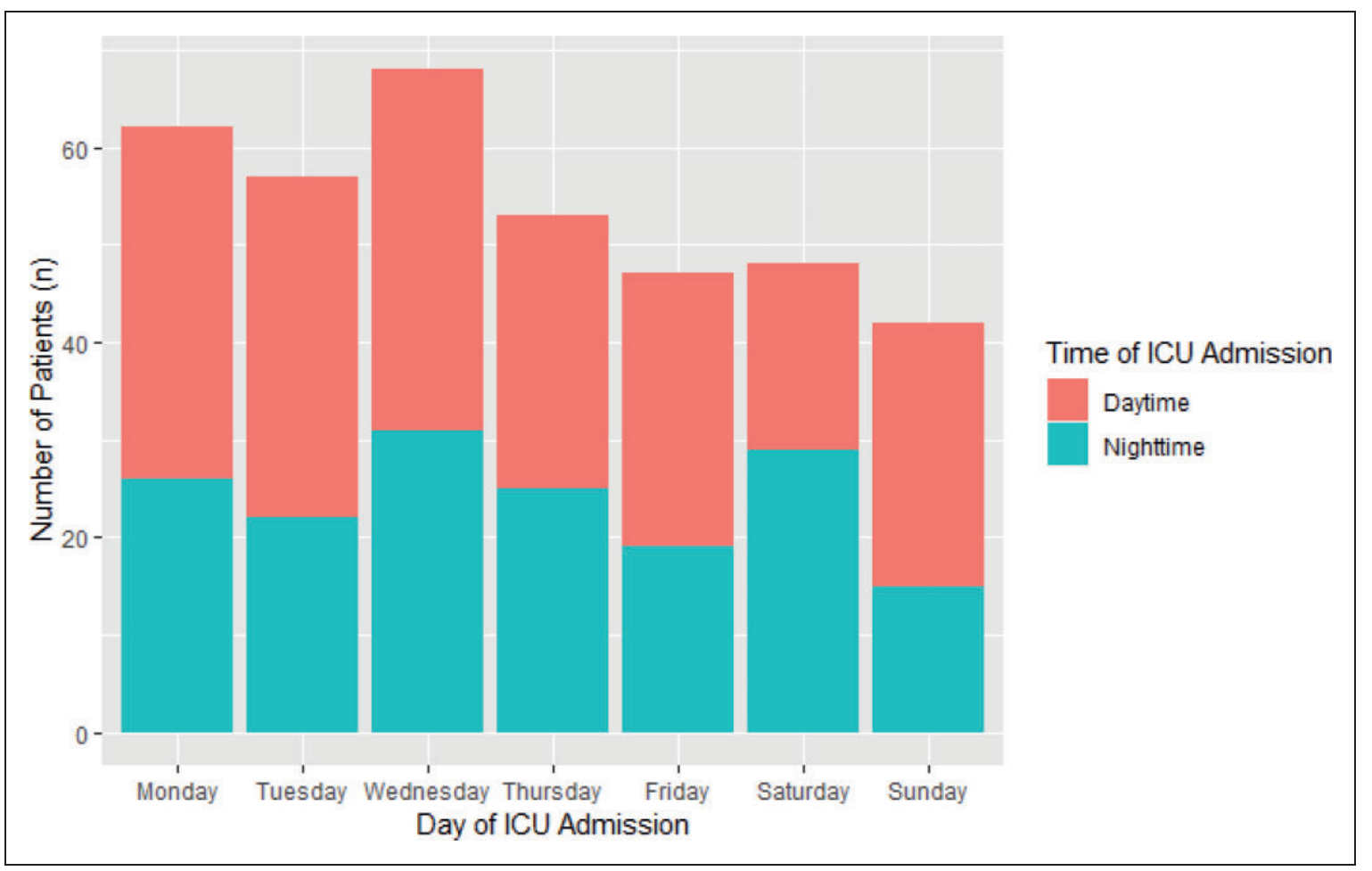

Figure 2. Histogram of ICU admissions stratified by time of ICU admission. ICU, intensive care unit. 


\section{Results}

During the study period, 499 patients were admitted to the study ICU and 377 met the inclusion criteria (Figure 1). The multivariable analyses included 346 patients after accounting for missing data. The cohort was $52 \%$ female (median age $=31$ years; IQR $=23-41$ years) (Table 1). Patients were admitted with multiple admission diagnoses; the most common were respiratory failure, severe head injury, postoperative monitoring and haemorrhagic shock (Supplementary materials, Tables S1-S2). The proportion of patients admitted during DT versus NT for each day of the week is summarised in Figure 2.

\section{Time of day}

There were $210(55.7 \%)$ DT admission versus 167 (44.3\%) NT admissions. Patients admitted during DT were more likely to have a clinically suspected infection $(62.4 \%$ vs. $45.5 \%, P=0.0015)$, while patients admitted at NT were more likely to have had surgery preceding admission to the ICU (69.5\% vs. $58.1 \%, P=0.0304)$. There were no other differences between the groups. Hospital mortality for DT admissions was $56.2 \%$ versus $57.5 \%$ for NT admissions $(P=0.8828)$ (Table 1). In the univariate survival analysis, there was no difference in the hospital mortality for patients admitted to the ICU during the DT versus the NT $(P=0.650)$ (Figure 3) and these results were unchanged in the multivariable Cox proportional hazards model $(\mathrm{HR}=1.09$, 95\% CI $=0.82-1.45, P=0.5614$; Figure 5 and Table $\mathrm{S} 4$ in Supplementary Materials) and in the supplementary analyses excluding patients with head injuries (Supplementary Materials). No significant interaction between day of the week and time of day was found (Supplementary Materials).

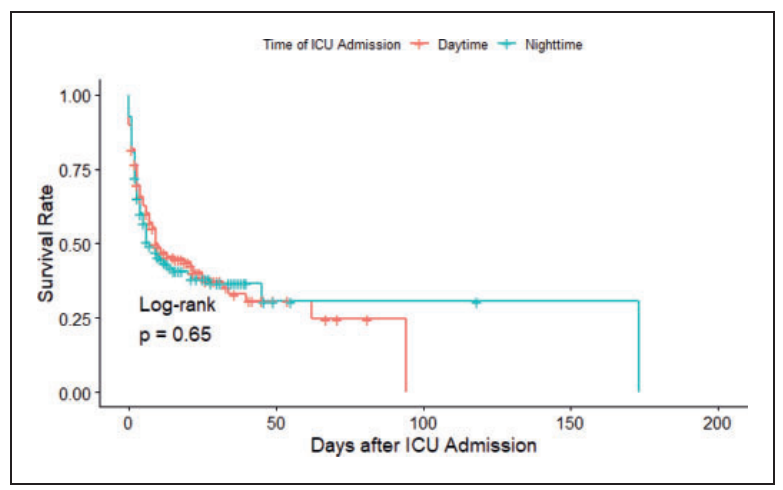

Figure 3. Unadjusted Kaplan-Meier curve for survival of daytime vs. night-time ICU admissions. ICU, intensive care unit.

\section{Day of the week}

There were $273(72.4 \%)$ weekday admissions versus 104 $(27.6 \%)$ weekend or public holiday admissions. Weekday admissions were more often general surgery patients $(63.7 \%$ vs. $57.7 \%)$ or medicine $(13.6 \%$ vs. $7.7 \%$ ) and weekend/holiday admissions were more often obstetrics patients $(34.6 \%$ vs. $22.7 \%)$. There were no other major differences. Hospital mortality was $57.1 \%$ for weekday admissions versus $55.8 \%$ for weekend or public holiday admissions $(P=0.9011)$ (Table 2). In the univariate survival analysis, there was no difference in the hospital survival for patients admitted to ICU during the week versus the weekend or public holidays $(P=0.95)$ (Figure 4); these results were unchanged in the multivariable Cox proportional hazards model $(\mathrm{HR}=0.87,95 \%$ $\mathrm{CI}=0.62-1.21, P=0.4133$; Figure 6 and Table S6 in Supplementary Materials) and in the supplementary analyses excluding patients with head injuries (Supplementary Materials).

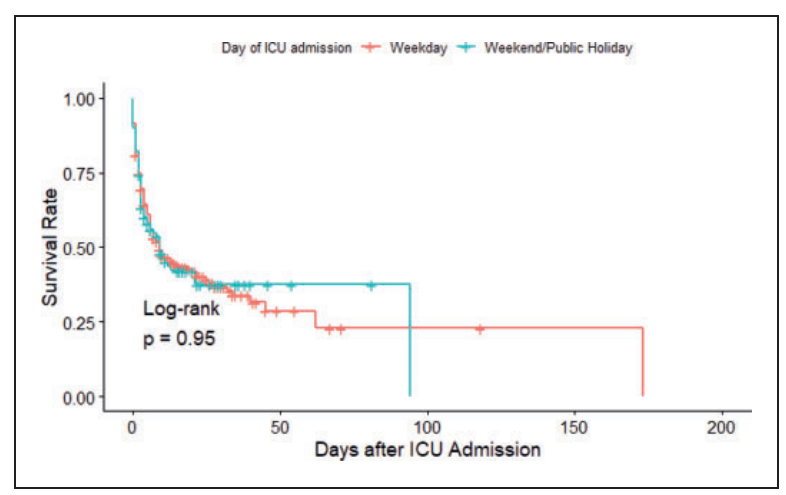

Figure 4. Unadjusted Kaplan-Meier curve for survival of weekday vs. weekend/holiday ICU admissions. ICU, intensive care unit.

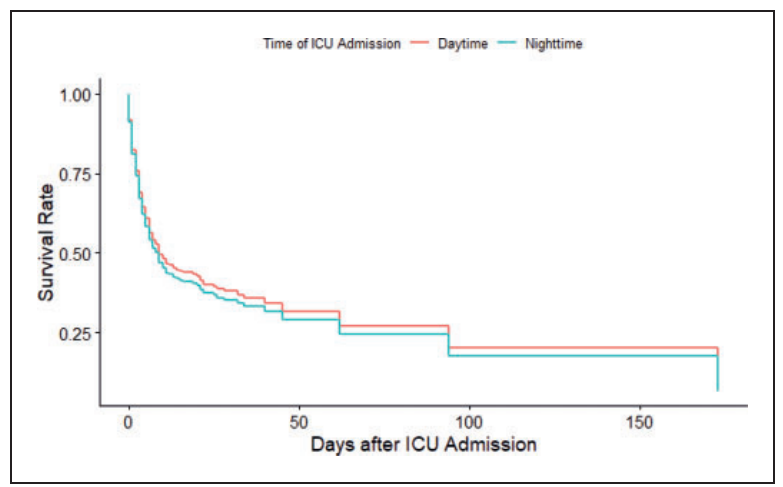

Figure 5. Adjusted Kaplan-Meier curve for survival of daytime vs. night-time ICU admissions. ICU, intensive care unit. 
Table 2. Clinical characteristics and hospital course for study cohort, stratified by day of the week of ICU admission.

\begin{tabular}{|c|c|c|c|c|}
\hline Variable & Total & Weekday & Weekend/ holiday & $P$ value \\
\hline Total ICU admissions & 377 & $273(72.4)$ & $104(27.6)$ & \\
\hline Age (years) & $3 I(23-4 I)$ & $31(23-42)$ & $33(23-40)$ & $0.7526 *$ \\
\hline Sex & & & & $0.138^{\dagger}$ \\
\hline Female & $196(52.0)$ & $135(49.5)$ & $6 \mathrm{I}(58.7)$ & \\
\hline Male & $|8|(48.0)$ & $138(50.5)$ & $43(4 \mid .3)$ & \\
\hline \multicolumn{5}{|c|}{ Hospital course preceding ICU admission } \\
\hline Time in hospital before ICU (days) & I (0-2) & I (0-2) & I (0-I) & $0.1509 *$ \\
\hline Hospital location before ICU admission & & & & $0.751^{+}$ \\
\hline Emergency room & $71(18.8)$ & $53(19.4)$ & $18(17.3)$ & \\
\hline HDU & $91(24.1)$ & $65(23.8)$ & $26(25.0)$ & \\
\hline Operating room & $180(47.7)$ & $132(48.4)$ & $48(46.2)$ & \\
\hline Outside hospital & $12(3.2)$ & $9(3.3)$ & $3(2.9)$ & \\
\hline Ward & $23(6.1)$ & $14(5.1)$ & $9(8.7)$ & \\
\hline Recent surgery in hospital course & $238(63.1)$ & $17 \mid(62.6)$ & $67(64.4)$ & $0.8401^{\dagger}$ \\
\hline Cardiac arrest before ICU & 45 (1 I.9) & $33(12.1)$ & $12(11.5)$ & $\sim 1^{\dagger}$ \\
\hline ICU admitting service & & & & $0.0348^{\dagger}$ \\
\hline General surgery & $234(62.1)$ & $174(63.7)$ & $60(57.7)$ & \\
\hline OBGyn & $98(26.0)$ & $62(22.7)$ & $36(34.6)$ & \\
\hline Medicine & $45(11.9)$ & $37(13.6)$ & $8(7.7)$ & \\
\hline \multicolumn{5}{|c|}{ Vital signs and assessments at admission to ICU } \\
\hline Total MEWS & $7(5-8)$ & $7(5-8)$ & $7(5-9)$ & $0.5418^{*}$ \\
\hline Heart rate (bpm) & $117(98-134)$ & 116 (95.75-132) & $119(104.25-142.75)$ & $0.03543 *$ \\
\hline Respiratory rate & $19(14-25)$ & $18(14-24)$ & $20(15-28.5)$ & $0.1055^{*}$ \\
\hline MAP $(\mathrm{mmHg})$ & 88.77 (72.27-104.86) & 88.77 (7I.28-106.18) & 91.25 (73.10-103.04) & $0.8528 *$ \\
\hline Temperature $\left({ }^{\circ} \mathrm{C}\right)$ & $36(34.7-37)$ & $36(34.68-37)$ & $36(34.7-37)$ & $0.4449 *$ \\
\hline Pulse oximetry value & 97 (93-99) & $97(92-99)$ & 98 (94.75-99) & $0.6192 *$ \\
\hline Altered mental status & $313(83.7)$ & $230(84.6)$ & $83(8 \mathrm{I} .4)$ & $0.558 \mathrm{I}^{\dagger}$ \\
\hline Infection suspected & $207(54.9)$ & $145(53.1)$ & $62(59.6)$ & $0.3086^{\dagger}$ \\
\hline HIV-positive & $4 \mid(12.9)$ & $26(11.3)$ & $15(17.2)$ & $0.2232^{\dagger}$ \\
\hline Malaria & $33(11.5)$ & $23(10.8)$ & $10(13.2)$ & $0.7397^{\dagger}$ \\
\hline \multicolumn{5}{|l|}{ Clinical course in ICU } \\
\hline Provision of mechanical ventilation & $364(96.6)$ & $265(97.1)$ & $99(95.2)$ & 0.3584 \\
\hline Length of mechanical ventilation (days) & $2(I-6)$ & $2(I-6)$ & $2(I-6)$ & $0.8018^{*}$ \\
\hline Length of stay in ICU (days) & $3(1-6)$ & $3(I-6)$ & $3(1-7)$ & $0.908 *$ \\
\hline ICU mortality & $191(50.7)$ & 137 (50.2) & $54(51.9)$ & $0.8518^{\dagger}$ \\
\hline Hospital mortality & $214(56.8)$ & $156(57.1)$ & $58(55.8)$ & $0.901 \mathrm{I}^{\dagger}$ \\
\hline
\end{tabular}

Values are given as $\mathrm{n}(\%)$ or median (IQR).

*Wilcoxon rank-sum test (non-parametric equivalent for two-sample $t$ test) for comparisons of continuous variables.

${ }^{\dagger}$ Chi-squared test for homogeneity/independence of categorical variables: Assumptions: (i) independent groups; (ii) no expected cell counts are 0 and no more than $20 \%$ of cells have an expected count $<5$.

"Fisher's exact test for count data in hybrid model: For tables larger than $2 \times 2$, in hybrid model, asymptotic Chi-squared probabilities are only used if the "Cochran conditions" (i.e. no expected cell counts are 0 and no more than $20 \%$ of cells have an expected count $<5$ ) are satisfied, otherwise the exact calculation is used. (In the $r \times c$ case with $r>2$ or $c>2$, if tables are too large for the exact test, Fisher's exact test for count data with simulated $P$ value [based on 2000 replicates] can be used as well.)

$\S \mathrm{MAP}$ is calculated using the following equation: $0.66 \times($ diastolic BP) $+0.33 \times($ systolic $B P)$.

BP, blood pressure; bpm, beats per minute; ICU, Intensive Care Unit; IQR, interquartile range; HDU, high-dependency unit; HIV, human immunodeficiency virus; MAP, mean arterial pressure; MEWS, Modified Early Warning Score; OBGyn, Obstetrics and Gynaecology. 


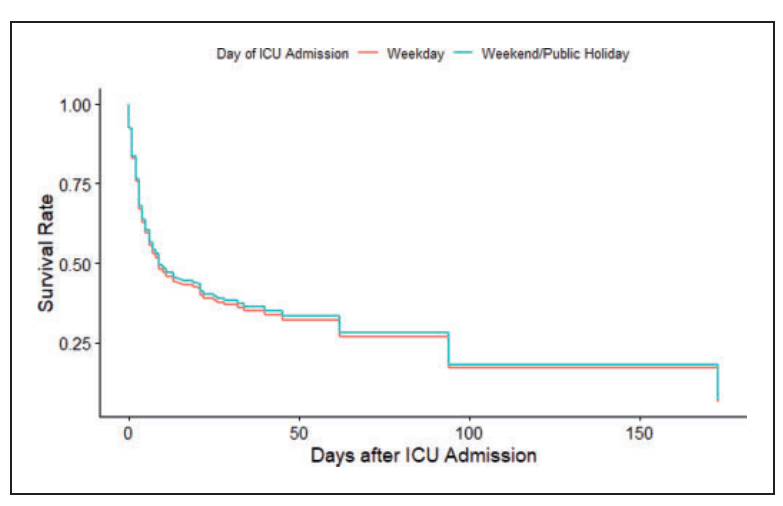

Figure 6. Adjusted Kaplan-Meier curve for survival of weekday vs. weekend/holiday ICU admissions. ICU, intensive care unit.

\section{Discussion}

Our study demonstrates that day of the week and time of day admission to the ICU have no association with hospital mortality in a critically ill cohort at a referral hospital in Malawi. While our findings regarding DT versus NT are consistent with studies from high-income nations, ${ }^{5,14}$ we did not find evidence of the "weekend effect' which has been demonstrated in high-income settings. ${ }^{14,20}$ This may be attributable to the high overall hospital mortality in this cohort, although we cannot rule out the possibility of a type 2 error given our relatively small sample size. Low-income countries face infrastructural challenges in the provision of healthcare, and studies such as ours which evaluate the effects of different organisational strategies may contribute to improved care delivery. A major strength of this study is the granularity of data collected within a region in which data collection is known to be difficult, and our findings contribute to the literature gap in characterising the processes of critical care services and outcomes in sub-Saharan Africa.

An important distinction between this study cohort and those from high-income settings is the overall hospital mortality. Hospital mortality in this study was $56.8 \%$ compared to overall crude hospital mortality generally $<20 \%$ in the studies included in the above referenced systematic review. ${ }^{14}$ This high hospital mortality for critically ill patients in Malawi has been described in our previous work ${ }^{19}$ and reflects the context of critical care medicine in this region. In an ICU setting with no physician oversight and supply shortages, differences between DT/NT or weekday/weekend staffing models may be less pronounced. Furthermore, our study cohort was predominantly surgical $(63.1 \%)$. Intraoperative patients who develop critical illness are moving from one high-intensity setting (operating theatre) to another (ICU), regardless of the time of the day or the day of the week. Our results may have differed if this study focused exclusively on patients who developed critical illness and were admitted to ICU from general wards and/or the emergency ward, where staffing patterns may have higher variance.

Nevertheless, our study provides an opportunity to evaluate how critically ill patients may be triaged in a setting of extreme scarcity. With only 1.7 ICU beds per million population in Malawi, ICU care must be reserved for those who are most likely to benefit ${ }^{21}$ and staffing models must be optimised based on what is known about ICU admission patterns. Our study found some differences in the clinical characteristics for patients admitted at different times. Off-hours (weekends, holidays and NT) admissions were more likely to be obstetric patients. This may indicate the higher incidence of obstetric emergencies in the offhours. Given that obstetric utilisation of ICU beds in Malawi is nearly one in four, ${ }^{22}$ hospital and ICU management personnel may plan, for example, to preferentially staff the ICU with obstetric specialists in the off-hours. We recognise that staffing in lowincome areas is often too short for this type of customisation and do not mean to oversimplify these issues; these data are meant to inform hospital resource allocation as it improves.

Identification of infrastructural factors affecting patient outcomes is an important component of quality improvement. Nevertheless, this study has several limitations. First, our study was conducted at a single site in a relatively small ICU, which limited the sample size. Second, we measured the time of admission to the ICU but not the time at which patients were recommended for admission. Variations in the delay between ICU referral and admission could have affected the clinical presentation at ICU admission. We conducted multivariable adjustment using a model with discrimination for ICU and hospital mortality in Malawi ${ }^{19}$ to adjust for these clinical differences. We were also unable to differentiate between altered mental status at ICU admission secondary to disease pathology and altered mental status secondary to the residual effects of anaesthesia in this predominantly surgical population. Finally we assumed that the relevant staffing models for weekdays, weekends and the time of day were consistent throughout the study period.

\section{Conclusion}

Our prospective cohort study demonstrates that the majority of ICU admissions at a referral hospital in Malawi occur from Monday to Friday and during DT working hours. We found high overall hospital mortality, but no difference in crude or adjusted hospital mortality based on the timing of ICU admission. These results may be affected by the high overall 
hospital mortality and resource restrictions in Malawi. Nevertheless, they provide an initial assessment of ICU admission patterns and may inform staffing models.

\section{Declaration of conflicting interests}

The author(s) declared no potential conflicts of interest with respect to the research, authorship, and/or publication of this article.

\section{Funding}

The author(s) received the following financial support for the research, authorship, and/or publication of this article: This study was supported in part by the NIH Fogarty International Center Postdoctoral Research Fellowship to MP. The contents of the manuscript are solely the responsibility of the authors and do not necessarily reflect the official views of the funding agencies.

\section{Supplemental material}

Supplemental material for this article is available online.

\section{ORCID iD}

Meghan Prin (D) https://orcid.org/0000-0002-2886-4666

\section{References}

1. Garland A, Roberts D and Graff L. Twenty-four-hour intensivist presence: a pilot study of effects on intensive care unit patients, families, doctors, and nurses. Am J Respir Crit Care Med 2012; 185: 738-743.

2. van der Sluijs A, van Slobbe-Bijilsma E, Chick S, et al. The impact of changes in intensive care organization on patient outcome and cost-effectiveness-a narrative review. J Intensive Care 2017; 5: 13.

3. Carson S, Stocking C, Podsadecki T, et al. Effects of organizational change in the medical intensive care unit of a teaching hospital: a comparison of 'open' and 'closed' formats. JAMA 1996; 276: 322-328.

4. Kane R, Shamiliyan T, Mueller C, et al. The association of registered nurse staffing levels and patient outcomes: systematic review and meta-analysis. Med Care 2007; 45: 1195-1204.

5. Arulkumaran N, Harrison D and Brett S. Association between day and time of admission to critical care and acute hospital outcome for unplanned admissions to adult general critical care units: cohort study exploring the 'weekend effect'. Br J Anaesth 2017; 118: 112-122.

6. Ju M, Tu G, Han Y, et al. Effect of admission time on mortality in an intensive care unit in Mainland China: a propensity score matching analysis. Crit Care 2013; 17: R230.

7. Hall AM, Stelfox HT, Wang X, et al. Association between afterhours admission to the intensive care unit, strained capacity, and mortality: a retrospective cohort study. Crit Care 2018; 22: 97.

8. Barnett MJ, Kaboli PJ, Sirio CA, et al. Day of the week of intensive care admission and patient outcomes: a multisite regional evaluation. Med Care 2002; 40: 530-539.

9. Uusaro A, Kari A and Ruokonen E. The effects of ICU admission and discharge times on mortality in Finland. Intensive Care Med 2003; 29: 2144-2148.

10. Laupland KB, Shahpori R, Kirkpatrick AW, et al. Hospital mortality among adults admitted to and discharged from intensive care on weekends and evenings. $J$ Crit Care 2008; 23: 317-324.

11. Cavallazzi R. Association between time of admission to the ICU and mortality: a systematic review and metaanalysis. Chest 2010; 138: 68.

12. Diaz JV, Riviello ED, Papali A, et al. Global Critical Care: Moving Forward in Resource-Limited Settings. Ann Glob Health 2019; 85: 3.

13. Murthy S, Leligdowicz A and Adhikari N. Intensive Care Unit Capacity in Low-Income Countries: A Systematic Review. PLoS One 2015; 10: e0116949.

14. Galloway M, Hegarty A, McGill S, et al. The effect of ICU out-of-hours admission on mortality: a systematic review and meta-analysis. Crit Care Med 2018; 46: 290-299.

15. Agha R, Borrelli M, Vella-Baldacchino $M$, et al. The STROCSS statement: strengthening the reporting of cohort studies in surgery. Int J Surg 2017; 46: 198-202.

16. United Nations. Human Development Reports. United Nations Development Programme. New York, NY: United Nations, 2016.

17. Gregson J. The Richest Countries in the World. International Monetary Fund, World Economic Outlook Database. 2018. Available at: https://www. gfmag.com/global-data/economic-data/richest-countriesin-the-world.

18. Prin M, Quinsey C, Kadyaudzu C, et al. Brain death in low-income countries: a report from Malawi. Trop Doct 2019; 49: 107-112.

19. Prin M, Pan S, Kadyaudzu C, et al. Development of a Malawi Intensive care Mortality risk Evaluation (MIME) model, a prospective cohort study. Int J Surg 2018; 60: 60-66.

20. Honeyford K, Cecil E, Lo M, et al. The weekend effect: does hospital mortality differ by day of the week? A systematic review and meta-analysis. BMC Health Serv Res 2018; 18: 870.

21. Manda-Taylor L, Mndolo S and Baker T. Critical care in Malawi: The ethics of beneficence and justice. Malawi Med J 2017; 29: 268-271.

22. Prin M, Kadyaudzu C, Aagaard K, et al. Obstetric admissions and outcomes in an intensive care unit in Malawi. Int J Obstet Anesth 2019; 39: 99-104. 